\title{
Biological Evidence and Molecular Modeling of a Grapevine Pinot gris Virus Outbreak in a Vineyard
}

\author{
Jean-Michel Hily, ${ }^{1, \dagger}$ Véronique Komar, ${ }^{2}$ Nils Poulicard, ${ }^{3}$ Emmanuelle Vigne, ${ }^{2}$ Olivier Jacquet, ${ }^{4}$ Nathalie Protet, ${ }^{4}$ \\ Anne-Sophie Spilmont, ${ }^{1}$ and Olivier Lemaire ${ }^{2}$ \\ ${ }^{1}$ Institut Français de la Vigne et du Vin (IFV), Le Grau-Du-Roi, France \\ ${ }^{2}$ Université de Strasbourg, Institut National de Recherche pour l'Agriculture, l'Alimentation et l'Environnement (INRAE), SVQV UMR-A 1131, \\ F-68000 Colmar, France \\ ${ }^{3}$ PHIM Plant Health Institute, Université de Montpellier, IRD, CIRAD, INRAE, Institut Agro, Montpellier, France \\ ${ }^{4}$ Chambre d'Agriculture du Vaucluse (CA84), Orange, France
}

Accepted for publication 26 April 2021.

\section{ABSTRACT}

Since its identification in 2003, little has been revealed about the spread of grapevine Pinot gris virus (GPGV), an emerging grapevine virus. According to studies from Italy, GPGV transmission in the vineyard can be fast but progressive over the years. To gain new insights into the spread of GPGV infections, we tested 67 grapevines in a single vineyard parcel in southern France. These vines were sampled over 8 years (2013 to 2020) and tested for GPGV by reverse-transcription PCR using a new primer pair designed from the recently described genetic diversity of GPGV worldwide. While focusing on a portion of the samples $(n=20)$, we observed a drastic increase in newly GPGV-infected vines from 2014 (5\%, 1 of 20$)$ to 2015 (80\%, 16 of 20 ) and $2016(90 \%, 18$ of 20$)$. Infected vines were scattered throughout the vineyard with no distinct pattern of distribution, and some rare vines remained negative through 2020. Using all available genomic information, we performed Bayesian-based phylogeographic analyses that identified a major intravineyard transmission in 2014 to 2015 . To test our model, we analyzed 47 additional grapevines and confirmed the outbreak of GPGV in 2015 , validating our in silico projection. Interestingly, some grapevines remained negative throughout the study, in spite of their close proximity to infected plants. These results raise questions about the dynamics of vector populations and environmental conditions that may be required for virus spread to occur in the vineyard.

Keywords: detection, GPGV, grapevine, phylodynamic modeling, spatiotemporal infection spread

\section{†Corresponding author: J.-M. Hily; jean-michel.hily@vignevin.com}

Author contributions: Conceptualization by J.M.H. and E.V.; methodology and investigation by J.M.H., V.K., N.Po., O.J., and N.Pr.; software by J.M.H. and N.Po.; writing, original draft by J.M.H.; writing, review, and editing by all authors; funding by J.M.H., E.V., O.L., and A.S.S.; and supervision by O.L. and A.S.S

Funding: This work was supported by INRAE and by IFV and by the projects VACCIVINE and GPGV funded through Plan National Dépérissement du vignoble (French Ministry of Agriculture) in 2017 and 2019, respectively. A fellowship from Moët \& Chandon, Comité Interprofessionnel du vin de Champagne, Bureau Interprofessionnel des Vins de Bourgogne, and Comité Interprofessionnel des Vins d'Alsace was awarded to J.-M. Hily.

*The $\boldsymbol{e}$-Xtra logo stands for "electronic extra" and indicates that supplementary tables and supplementary figures are published online.

The author(s) declare no conflict of interest.

(c) 2021 The American Phytopathological Society
Grapevine is a crop that can be infected by a large number of viral species (Fuchs 2020; Martelli 2017). With the advent of high-throughput sequencing (HTS) techniques, many new grapevine-infecting viruses are being identified (Bertazzon et al. 2020a; Diaz-Lara et al. 2019). Grapevine Pinot gris virus (GPGV) is a newly emerging virus that was identified by HTS for the first time in grapevines from the northeastern region of Italy (Giampetruzzi et al. 2012). GPGV belongs to the genus Trichovirus from the family Betaflexiviridae. Its genome consists of a single positive-sense polyadenylated RNA molecule that is 7,259 nucleotides (nt) in length. The virus is associated with a disease known as grapevine leaf mottling and deformation (GLMD). Many symptoms may be associated with GLMD with numerous confounding factors, ranging from biotic to abiotic stresses, such as coinfection with other pathogens (viral, bacterial, or fungal) or adverse environmental conditions. Typical symptoms are generally clearly visible in the early stages of plant development, with partial to complete recovery during the growing season. Most of the grapevines infected with GPGV are symptomless. Koch's postulates have yet to be completely fulfilled to 
ascertain the etiology of GLMD (Tarquini et al. 2019b). In addition, a correlation between the occurrence of the disease and specific "symptomatic" GPGV variants (Saldarelli et al. 2015) remains debatable, with many studies challenging this concept (Bertazzon et al. 2017, 2020b; Marra et al. 2020; Renault-Spilmont et al. 2018).

Since its discovery, GPGV has been detected in almost every grape-growing region around the world, starting with the first reports in European wine grape production regions (Beuve et al. 2015; Pleško et al. 2014; Reynard et al. 2016; Ruiz-García and Olmos 2017) to other grapevine-growing countries (Al Rwahnih et al. 2016; Fajardo et al. 2017; Poojari et al. 2016; Wu and Habili 2017). The widespread occurrence of this virus raises questions about its dissemination. A recent study based on the analyses of sequences obtained via data mining showed that different lineages of GPGV display distinct evolutionary characteristics (Hily et al. 2020). After its probable emergence in Asia in the mid- to late 19th century, the virus spread through Europe, which subsequently served as a hub for distribution to the rest of the world. This worldwide movement of the virus certainly occurred via propagation and uncontrolled distribution of infected material, as previously suggested (Fajardo et al. 2017; Wu and Habili 2017). Additionally, it has been demonstrated that the virus can be transmitted by grafting (Saldarelli et al. 2015). Within vineyards, the virus was suggested to be transmitted by a vector on the basis of aggregated patterns of GLMD symptomatic vines (Bertazzon et al. 2015; Malossini et al. 2015). Recently, the spread of the virus was confirmed in two vineyards in the Veneto region of Italy (Bertazzon et al. 2020b). Colomerus vitis (Acari; Eriophyidae) is thought to be the main vector, as has been shown for grapevine berry inner necrosis virus (GINV, Trichovirus) (Kunugi et al. 2000), a close relative to GPGV. However, the role of this monophagous eriophyid mite of grapevine in GPGV transmission has been confirmed in only semicontrolled conditions (Malagnini et al. 2016). Unlike GINV, which is restricted to grapevine, GPGV has been detected in woody and herbaceous hosts (Demián et al. 2018; Gualandri et al. 2017), suggesting that $C$. vitis may not be the sole vector of the virus. To better develop efficient management strategies to control the spread of GPGV, knowledge of its biology, epidemiology, genetic diversity, evolution, and ecology is needed. To date, information on disease epidemiology is scarce.

In this study, we document and provide insights into transmission events in a vineyard in France using phylodynamic reconstruction.

\section{MATERIALS AND METHODS}

Plant material and RNA extraction. The vineyard selected for this study was planted with Vitis vinifera 'Grenache' grafted onto rootstock 110R in 2003 in the southeastern wine region of Châteauneuf-du-Pape (CDP) in France. The vineyard, an experimental parcel dedicated to a grapevine fanleaf virus (GFLV) crossprotection assay, consisted of 623 vines distributed over 13 rows of approximately 55 vines per row. Prior to this work, most of the vines were shown to be positive for GFLV by enzyme-linked immunosorbent assay (ELISA) (data not shown). Due to the presence of GFLV and its possible confounding effect, symptoms of GPGV were not monitored in this study.

To determine the natural spread of GPGV, we focused our effort on 67 grapevines that were already assessed yearly (from 2013 to 2020) in the spring season (from late May to early June) for the presence of GFLV. Samples consisted of young apical leaves collected from multiple locations in the grapevine canopy to maximize viral detection. Leaf samples were then kept at $-20^{\circ} \mathrm{C}$ prior to downstream analyses.

Total RNA extraction was performed from $200 \mathrm{mg}$ of leaf tissue homogenized into Bioreba extraction bags (Bioreba AG, Reinach, Switzerland). RNA was then extracted using the RNeasy Plant
Mini Kit (Qiagen, Venlo, The Netherlands), as previously described (Vigne et al. 2018).

Reverse-transcription PCR. GPGV detection was performed following a two-step protocol. Briefly, cDNA was synthesized using SuperScript III (Invitrogen, Carlsbad, CA, U.S.A.) with a mix of oligo dT in a final volume of $20 \mu \mathrm{l}$, as per the manufacturer's recommendations, and kept at $-20^{\circ} \mathrm{C}$ prior to additional analyses. PCR was then carried out in a total volume of $50 \mu \mathrm{l}$, including $1 \mu \mathrm{l}$ of cDNA, $0.5 \mu \mathrm{l}$ of GoTaq (Promega Corp., Madison, WI, U.S.A.), and $0.4 \mu \mathrm{M}$ each newly designed GPGV detection primer (see dedicated section in the main text). The PCR cycling parameters were as follows: initial denaturation at $95^{\circ} \mathrm{C}$ for $2 \mathrm{~min}$, followed by 38 cycles of $30 \mathrm{~s}$ at $95^{\circ} \mathrm{C}, 30 \mathrm{~s}$ at $57^{\circ} \mathrm{C}$, and $1 \mathrm{~min}$ at $72^{\circ} \mathrm{C}$. A final elongation step of $5 \mathrm{~min}$ at $72^{\circ} \mathrm{C}$ was added prior to resolving PCR products on a $2 \%$ agarose gel.

A second primer pair (Fwd-GPGV-CDP_5350: AGTTAGGTTCA TGGCTTGATTGGG and Rev-GPGV-CDP_6990: AAGCGATTC AGATTTTGAATCACCGA) was designed to determine the genetic diversity of GPGV in the experimental vineyard by producing a 1,640-bp amplicon, from which 1,528 bp was used for sequence analyses. The PCR cycling parameters were identical to those in the abovementioned protocol, except that the elongation duration was increased to $2 \mathrm{~min}$ and $20 \mathrm{~s}$ and a final elongation step of $10 \mathrm{~min}$ at $72^{\circ} \mathrm{C}$ was included. PCR products were subjected to direct Sanger sequencing (Genoscreen, Lille, France) using the second primer pair.

To check cDNA quality, all samples were tested for the presence of glyceraldehyde-3-phosphate dehydrogenase (GAPDH) housekeeping transcripts as previously described (Garcia et al. 2019).

HTS, sanitary status, and complete GPGV genome sequences. A subset of 20 samples from 2017 was selected to prepare cDNA libraries after poly-A selection. All steps were performed at the GeT-PlaGe Genotoul platform facility (Institut National de Recherche pour l'Agriculture, l'Alimentation et l'Environnement [INRAE], Toulouse, France) as previously described (Vigne et al. 2018). Briefly, the HTS approach of choice in this study was a paired-end $2 \times 150$ bp RNA sequencing (RNAseq) completed on a HiSeq 3000 (Illumina, San Diego, CA, U.S.A.) following the manufacturer's instructions. Analyses were performed using Workbench 12.0 software (CLC Genomics Workbench, Aarhus, Denmark). The sanitary status of the samples was assessed by (i) mapping reads to a collection of curated reference sequences of viruses known to infect grapevine and (ii) de novo assembly steps from which contigs were tested against GenBank reference sequences using BlastN/BlastX, as previously described (Hily et al. 2018).

GPGV contigs assembled de novo were further extended by multiple rounds of residual read mapping. The resulting sequences were previously described and submitted to GenBank (GenBank MN458417 to MN458441) (Hily et al. 2020).

Genetic diversity analyses. Differences in the nucleotide diversity of viral populations multiple sequence alignments and neighborjoining and maximum-likelihood (ML)-based phylogenetic trees were prepared using MEGAX software (Kumar et al. 2018). The best ML-fitted model was used, and nodes in phylogenetic trees and branch validity were assessed by bootstrap analyses (100 replicates).

Discrete phylogeographic analyses. The magnitude of the temporal signal in the GPGV datasets was first investigated with an exploratory linear regression approach. After confirming the absence of recombinant sequences in the datasets, we reconstructed ML phylogenetic trees based on 116 complete GPGV genome sequences (not considering untranslated regions [UTRs]), as previously described (Hily et al. 2020). A second analysis was performed on a selection of 101 partial GPGV sequences (between nucleotide positions 5,399 and 6,926), removing noninformative sequences from the 
analysis (i.e., highly similar GPGV sequences from the same region of the world, other than the CDP region). We used TempEst v1.5.1 (Rambaut et al. 2016) to regress phylogenetic root-to-tip distances against sampling date. Then, the significance of the temporal signal was also evaluated by a date randomization test. The mean rate and its $95 \%$ highest probability density (HPD) estimated with the observed sampled dates using the Bayesian evolutionary analysis sampling trees (BEAST) v1.8.2 package (Drummond et al. 2012) were compared with a null distribution obtained by randomly permutating the tip dates six times (Firth et al. 2010). As previously described (Duchêne et al. 2015; Murray et al. 2016), the criterion for a significant temporal signal was that the 95\% HPD for the rate estimate obtained with the observed sampled dates should not overlap with the 95\% HPD for the estimate obtained with randomized sampling times.

Bayesian phylogeographic inferences were performed using discrete diffusion approaches in BEAST v1.8.2 and the BEAGLE library (Ayres et al. 2011) to improve computational performance. The BEAST program uses Markov chain Monte Carlo (MCMC) integration to average all plausible evolutionary histories for the data, as reflected by the posterior probability. A Hasegawa-Kishino-Yano 85 (HKY85) substitution model was applied, with discretized $\Gamma$ and I distributions to model rate heterogeneity across sites and invariable site proportions, respectively. An uncorrelated relaxed molecular clock that models branch rate variation according to a log normal distribution (Drummond et al. 2006) was specified to accommodate among-lineage rate variation. The flexible nonparametric demographic skygrid prior was selected (Gill et al. 2012).

Discrete phylogeographic inferences were estimated between the field (20 individual plants from the experimental vineyard of the CDP vineyards) and worldwide (Asia, America, and Europe, excluding the sequences from the experimental parcel) levels using the continuous-time Markov chain process (Lemey et al. 2009) and with Bayesian stochastic search variable selection. This method (i) reconstructs the dispersion history between discrete locations, (ii) infers a posterior distribution of trees whose internal nodes are associated with an estimated ancestral date and location, and (iii) estimates the number (Markov jumps) of discrete state transitions in the GPGV evolutionary history (Minin and Suchard 2008a, b). MCMC analyses were run for at least 600 million generations, sampling every 100,000 th generation and discarding $10 \%$ of the iterations as the chain burn-in. The maximum clade credibility (MCC) tree was obtained with TreeAnnotator 1.8.2 (Drummond et al. 2012), and convergence and mixing properties (e.g., based on effective sample sizes $>200$ for the parameters) were inspected using Tracer 1.6 (http://tree.bio.ed.ac. uk/software/tracer).

\section{RESULTS}

Molecular diagnostics and the design of a new and more inclusive GPGV detection primer pair. Although the diversity of GPGV is quite limited compared with that of other newly described species of grapevine viruses (Nourinejhad Zarghani et al. 2018), the unbiased addition of many new sequences from a previous study (Hily et al. 2020) allowed us to evaluate the polyvalence of existing detection primers and their capacity to detect all GPGV isolates. An in silico analysis clearly showed the inability of three primer pairs designed to date (Bertazzon et al. 2016; Bianchi et al. 2015; Morán et al. 2018) to efficiently detect all GPGV variants (Supplementary Fig. S1). Although only a few detection primers or probes (three of the eight tested) showed multiple mismatches, with some mismatches being located at the $5^{\prime}$-terminal nucleotides, which may not affect their ability to bind, other mismatches affected the last four 3'-most nucleotides of the primer and probe. Considering a conservative rule of less than three mismatches, with no mismatches within the four $3^{\prime}$-ultimate nucleotides for a primer or probe with a particular virus isolate, none of the primer pairs or probes examined were likely to detect all isolates. In light of the latest sequences available (Hily et al. 2020), a new degenerate primer pair was designed that theoretically has, in silico, the ability to detect all GPGV isolates. The sequence of these primers is GPGVall_Fwd: GTRAAGATGGT TAAGTCTARATCTGG and GPGValllong-REV: GCACACA CYTGACGAAAAGTCAT, producing a 411-bp amplicon in reverse-transcription (RT)-PCR located at the genomic region of the movement protein (MP) and coat protein (CP) overlap (MP/CP).

Spatial and temporal propagation of GPGV in a vineyard in southern France. The presence of GPGV was first assessed in 20 grapevines from a vineyard parcel located in the southeastern wine region of CDP in France. In 2013, of the 20 plants selected for this study that were distributed throughout the parcel, only 1 tested positive for GPGV by RT-PCR (Fig. 1, 2013) using the newly designed primers. The following year, the same grapevine, Ma-A2-37, was once again the only GPGV-positive grapevine. In 2015, 15 additional grapevines became GPGV positive. Newly infected grapevines were randomly distributed and not near the first GPGV-infected plant, MaA2-37 (Fig. 1, 2015). In 2016, two new grapevines became GPGV positive, leading to $90 \%$ (18 of 20) of the plants testing positive for the virus. In 2017, no new grapevines became infected (Fig. 1, 2017). Additional analyses performed on the last two negative vines showed that they were still GPGV negative in the spring of 2020 (Supplementary Table S1), 8 years after the first identification of GPGV in the study vineyard.

GPGV variant identification and location within the parcel. To identify GPGV variants within our experimental vineyard, the aforementioned 20 grapevines sampled in the spring of 2017 were used for downstream RNAseq analyses. The background virome in all grapevines tested consisted of grapevine Rupestris stem pittingassociated virus (GRSPaV) and two viroids - namely, hop stunt viroid (HSVd) and grapevine yellow speckle viroid-1 (GYSVd-1) - in all of the plants tested (Supplementary Table S2). In addition, GFLV was detected in all vines, and grapevine Rupestris vein feathering virus (GRVFV) was present in one of the vines. No new viral species were detected in any of the selected plants. A perfect correlation was observed between GPGV detection by RT-PCR using the newly developed "universal" primers and RNAseq data, with the 18 positive grapevines detected by RT-PCR being the one from which GPGV full-length sequences were obtained by RNAseq. From the 18 positive samples, 25 complete genome sequences were assembled (Hily et al. 2020). From all pairwise comparisons, the percentage of nucleic acid identity within the parcel was $\geq 97.73 \%$. Basic genetic diversity analyses showed that the overall mean distance $(\pi)$ of the GPGV population of this particular vineyard was $\pi=0.015 \pm 0.001$, a value comparable with the overall mean distance obtained from all French isolates known to date (taking into account sequences from other regions of France, such as Alsace, Champagne, and Bordeaux) with $\pi=0.017 \pm 0.002$ (Hily et al. 2020). A phylogenetic analysis showed that all 25 sequences belonged to the previously described "European" lineage of GPGV (Fig. 2A), which is distinct from the "Asian" lineage (Hily et al. 2020). Nine different clades could be defined when considering a conservative but realistic parameter of $1 \%$ variability as the demarcation criterion for GPGV, knowing its genetic diversity (Hily et al. 2020). Interestingly, most of the grapevines (11 of 18) were infected with a single variant of GPGV (Supplementary Table S2; Fig. 2). Surprisingly, all seven coinfections displayed a different mixture of GPGV variants, generally belonging to different clades, except for two plants (namely, Ma-A9-21 and Ma-10-18), for which variants belonged to the same clade (Fig. 2). Interestingly, only two plants (Ma-3-26 and Ma-A8-12) were infected with the same variant (deep blue) as the one detected in the original GPGV-infected grapevine 
Ma-A2-37 in 2013 (Fig. 2B). However, these two plants were quite distant from Ma-A2-37, being located one to six rows and 11 to 25 grapevines away from the original GPGV-infected grapevine. This apparently random distribution of vines infected with distinct GPGV variants was supported by the fact that a given GPGV variant was rarely detected in GPGV-infected adjacent vines. Indeed, only seven grapevines (of the 16 contiguous possibilities) displayed a variant belonging to the same clade, with only two plants presenting the exact same combination of variants (Ma-A9-21 and Ma-A9-22). These results collectively suggested limited randomness within vineyard transmission of GPGV and major introduction events from outside sources.

Phylodynamic reconstruction of the GPGV introduction and transmission in the CDP vineyard. To reconstruct epidemiological phases in our experimental vineyard, we used all 25 complete genome sequences (without UTRs) obtained in 2017 from CDP and 91 from vineyards all over the world (Hily et al. 2020). We performed a discrete phylogenetic analysis using BEAST (Fig. 3). As discussed in our previous study (Hily et al. 2020), the presence of a temporal signal was not completely fulfilled with the date randomization tests implemented in BEAST (Supplementary Fig. S2). Hence, the following dates (but not the locations) proposed in this phylodynamic reconstruction model must be considered with caution. Reconstruction of the evolutionary history of GPGV identified nine independent introductions of the virus in CDP vineyards (Fig. 3A, indicated by the red branches in the MCC tree) (location probabilities associated with nodes of interest are listed in Supplementary Table S3). The period with the highest probability of GPGV introduction in the CDP vineyard (i.e., when most of the date intervals overlapped) (Fig. 3B) was estimated to be between 1998 and 2004.

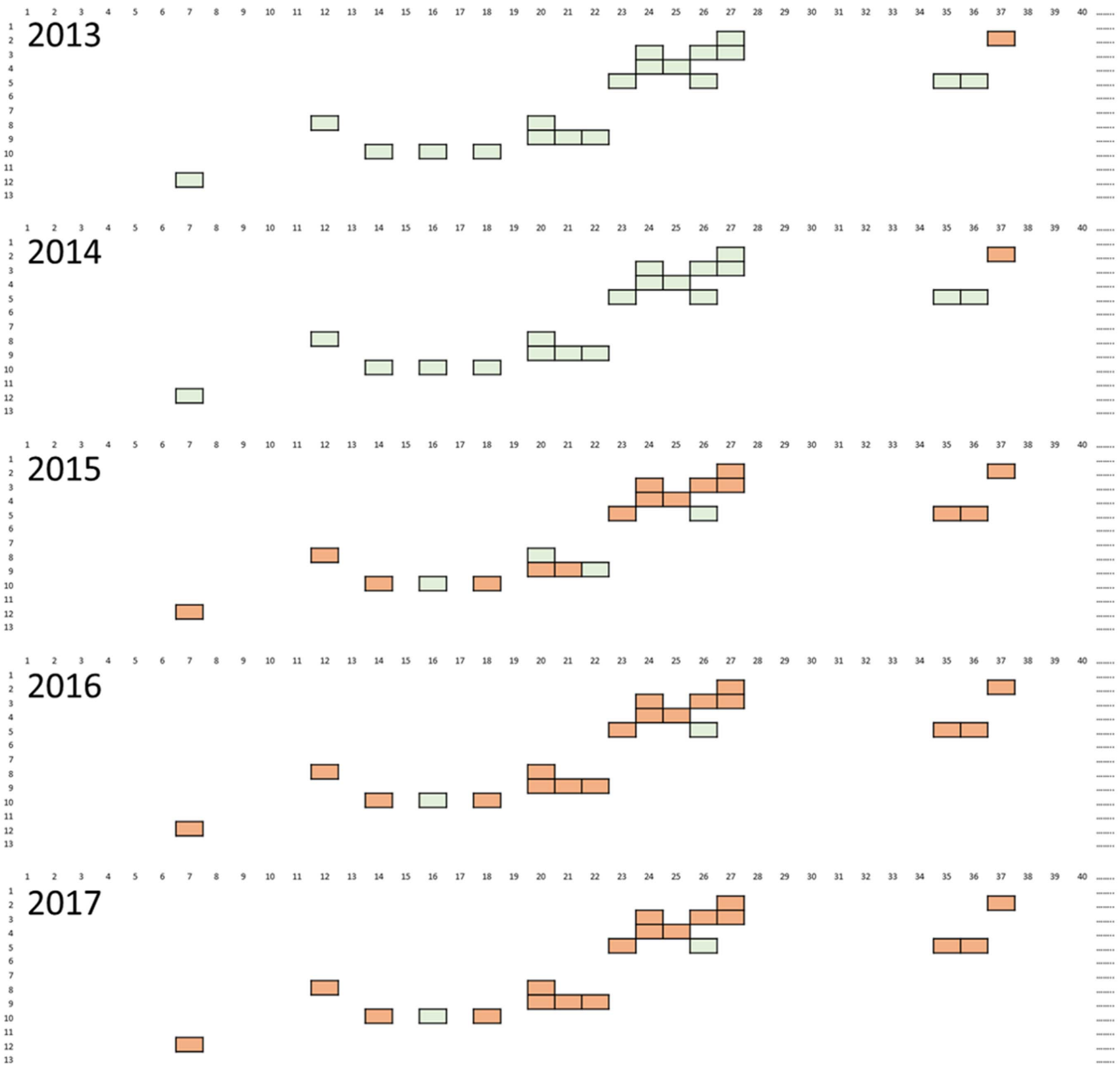

Fig. 1. Yearly evolution of grapevine Pinot gris virus (GPGV) infection status in 20 grapevines from a vineyard in the Châteauneuf-du-Pape region, France, from 2013 to 2017. Squares represent the vine locations in the parcel, and colors show the GPGV infection status, as determined by reversetranscription PCR (orange = positive and green = negative). 
Interestingly, while focusing on the GPGV transmission that occurred within the study vineyard in the CDP and other vineyards, the Bayesian reconstruction did not reveal intravineyard transmission prior to 2009 (Fig. 3C, lower limit of the 95\% HPD: 2004; Supplementary Table S3). In addition, the highest probability of GPGV transmission between plants within the experimental vineyard was estimated to occur in 2014 to 2015 (Fig. 3C; Supplementary Table S3).

Biological confirmation of the model projection. Based on the analyses of GPGV sequences from 20 grapevines, we observed a drastic increase in newly GPGV-infected vines in the study vineyard, with $75 \%$ of the plants becoming positive for the virus in 2015, which was also identified by phylodynamic reconstruction. To better comprehend the spread of the virus in the study vineyard parcel and possibly confirm the model projection, additional grapevines were tested for the presence of GPGV, focusing on 2014 and 2015. Forty-seven new plants that were distributed around the 20 grapevines that were previously analyzed were tested. In 2014, the virus was detected in 3 of 47 plants, corresponding to $6 \%$ of the total plants tested that year (4 of 67) (Fig. 4). In 2015, the number of positive cases (37 of 47) substantially increased and corresponded to $79 \%$ (53 of 67) of the plants tested. This result validated our initial findings based on 20 grapevines and the transmission outbreak estimated by our phylodynamic reconstruction model.

Furthermore, we checked the presence of the virus in the negative vines (14 of 67) from 2016 to 2020 (Supplementary Tables S1 and S4). As previously observed, only a marginal proportion of grapevines became infected after the outbreak. Only four new plants
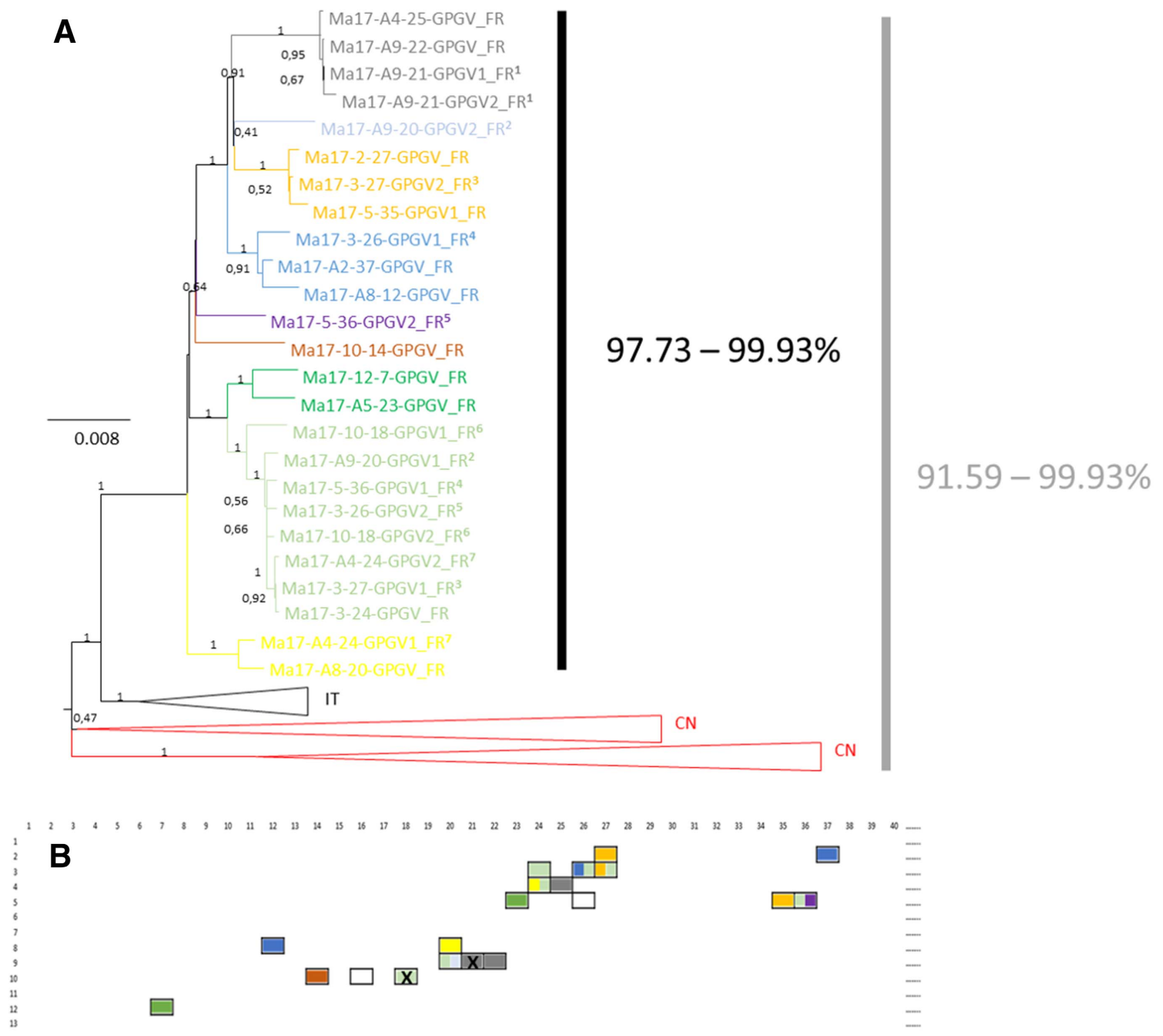

Fig. 2. Definition of variants in nine clades and their location in the parcel in 2017. A, Maximum-likelihood tree inferred from all complete genome sequences covering at least the different open reading frames of grapevine Pinot gris virus (GPGV) obtained from the 18 GPGV-positive samples and with other sequences representing the known genetic diversity in the world: European (Italian [IT]) and Chinese (CN) sequences. Colors correspond to a single sequence or group of sequences within a maximum of $1 \%$ diversity that defines a clade, and a numerical superscript indicates the number and variants detected in coinfection in the same plant. B, Spatial distribution of each variant within a plant and the parcel. Cells with two colors correspond to grapevines coinfected by two variants of the virus. Cells with an $\mathrm{X}$ indicate grapevines infected by two variants belonging to the same clade. Empty cells correspond to GPGV-negative grapevines. 
became positive for the virus during the 5 years after the GPGV outbreak, with two plants becoming positive in 2016, one in 2018, and one in 2019. Thus, in 2020, more than $12 \%$ of the grapevines that were negative in 2013 remained GPGV negative ( 8 of 65), although all of these grapevines were surrounded by GPGV-positive plants.

Genetic stability of the virus over the years and fine tuning of the model. To better understand virus evolution and variant movement within the vineyard, we focused our effort on 10 grapevines in which GPGV sequences were detected over time (Supplementary Table S5). We amplified and analyzed a section containing a 1,528-bp fragment of the virus genome. This MP/CP overlap region was the most divergent (Hily et al. 2020), allowing the discrimination of the nine abovementioned clades detected in our experimental parcel via HTS (Supplementary Fig. S3).

First, we compared sequencing techniques (that is, Illumina versus Sanger) by focusing on sequences obtained from the samples collected in 2017 (Supplementary Table S5). We observed an average of 1.25 mismatches per comparison throughout the complete amplicon sequence, corresponding to an error rate of $0.08 \%$ per site between sequencing techniques (Supplementary Table S5). It is worth mentioning that the direct Sanger sequencing process allowed the detection of a single variant in two grapevines (Ma-3-27 and Ma-A4-24) which were, according to HTS-based sequencing, infected by two distinct variants (Supplementary Table S2; Fig. 2A). For both grapevines, the variant detected by Sanger sequencing was the major variant identified by HTS (i.e., with higher reads per kilobase million [RPKM]) (data not shown).

We then tested the genetic stability of the virus in eight plants over the years by comparing Sanger sequences. The GPGV sequences were apparently very stable over time (Supplementary Table S5), with a maximum of seven mismatches, corresponding to $99.52 \%$ identity, well within the abovementioned $99 \%$ identity criterion for variant or clade demarcation. In addition, the major variant seemed to be the same 3 years in a row for the two multivariants infected grapevines (Supplementary Table S5, Ma-3-27 and Ma-A4-24).

These new partial genome sequences (and associated dates) were added to the previous global GPGV sequence dataset, and phylogenetic analyses were performed. As for the complete genome sequence dataset, the presence of a temporal signal in the partial genome sequence dataset was not confirmed with the date randomization tests

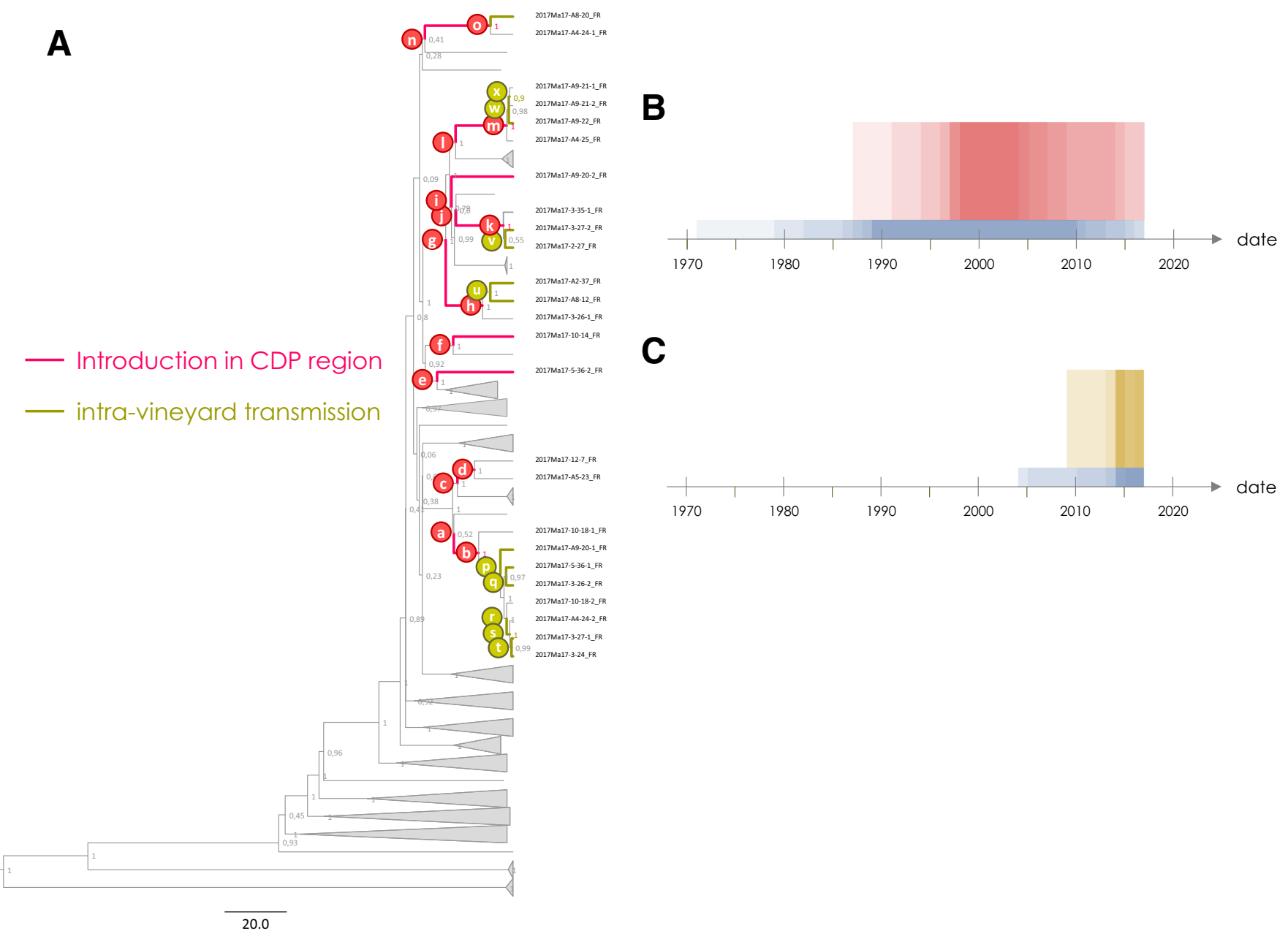

Fig. 3. Evolutionary history of grapevine Pinot gris virus (GPGV) focusing on our experimental vineyard. A, The maximum clade credibility tree was reconstructed from 116 full-length coding sequences of GPGV, with 25 sequences coming from the parcel itself. Values on the nodes correspond to their posterior probabilities. Twenty-four nodes of interest (from a to $\mathrm{x}$ ) were annotated with their time to the most common ancestor and with their location origin probability (Supplementary Table S3). Branches indicating introduction of GPGV in the Châteauneuf-du-Pape (CDP) region or intravineyard transmission are colored red and gold, respectively. B, Representation of the dates (in red) and the $95 \%$ highest probability density (HPD, in blue) intervals of the GPGV introductions in the CDP vineyards estimated as the intervals between nodes a and b, $c$ and $d, g$ and $h, j$ and $k$, $\mathrm{I}$ and $\mathrm{m}$, and $\mathrm{n}$ and $\mathrm{o}$ and between nodes $\mathrm{e}, \mathrm{f}$, and $\mathrm{i}$ and the date of sampling (2017) (A) (Supplementary Table S3). C, Representation of the dates (in gold) and the 95\% HPD (in blue) intervals of GPGV transmission within the CDP vineyard estimated as the intervals between nodes $r$ and $s$, and w and $x$, and between nodes o, p, q, t, u, v, and w and the date of sampling (2017) (A) (Supplementary Table S3). 
implemented in BEAST (Supplementary Fig. S2). Hence, although the dates estimated in this phylodynamic reconstruction must be considered with caution, the results obtained with the partial genome sequences confirmed our previous results (Supplementary Fig. S4). For example, the highest probability of GPGV introduction in the CDP vineyard was estimated between 1999 and 2003 (Supplementary Fig. S4C). Furthermore, the high probability of GPGV transmission within the CDP vineyard in 2014 to 2015 was confirmed (Supplementary Fig. S4E). Interestingly, this new sequence dataset allowed the identification of an additional period with a high probability of GPGV transmission within CDP vineyards that occurred in 2009 to 2010. The sequence variants identified in the study vineyard were unique, because they were not identified in our comprehensive GPGV sequence repository (Supplementary Fig. S4D, MarkovJumps $_{\text {CDP_Parcelt }>\text { World }}=0.38 ; 95 \%$ HPD [0.00 to 1.69]).

\section{DISCUSSION}

To date, little is known about the spread of GPGV, a newly emerging virus infecting grapevine (Giampetruzzi et al. 2012). Other than a study performed in Italy (Bertazzon et al. 2020b), limited information is available on the transmission dynamics of GPGV in vineyards. Here, we characterized the spread of the virus in a parcel located in southeastern France over eight growing seasons, starting 10 years postplanting.

Prior to this work, most vines in our vineyard of interest were shown to be positive for GFLV by ELISA (data not shown). The presence of GFLV was confirmed using RNAseq in a selection of 20 grapevines sampled in 2017 (Supplementary Table S2). Due to the presence of GFLV, the main causal agent of fanleaf degeneration, and its possible confounding or synergistic effects, symptoms due to GPGV were not monitored in this study. The presence of GPGV was detected in 18 of the 20 selected grapevines, allowing the reconstruction of 25 full-length genomes. Together with other complete
GPGV genome sequences, an alignment was performed that allowed the design of a more inclusive GPGV detection primer pair. Unlike other primers, this new pair of primers theoretically has the ability to detect, in silico, all of the isolates, regardless of the GPGV genetic diversity described to date. By testing the newly designed primers for the presence of GPGV on the same 20 grapevines, the RT-PCR results perfectly correlated with the RNAseq data, detecting variants that were theoretically not detected by other primer pairs (Bertazzon et al. 2016; Bianchi et al. 2015; Morán et al. 2018). To fully validate the new primers, more work is needed, using samples that carry variants from different regions of the world, especially those belonging to the previously described Asian lineage (Hily et al. 2020), which display the highest diversity.

We estimated the highest probability of GPGV introduction to the CDP region, probably through infected planting material, to have occurred around the 2000s, with possible introduction as early as the 1980s, approximately two decades prior to the setup of our study vineyard in 2003 (Fig. 3). Similar conclusions were drawn when using a different set of partial sequences, focusing on the most divergent region of the GPGV genome, corresponding to a 1,528-nt stretch at the MP/CP overlap (Hily et al. 2020; Saldarelli et al. 2015; Tarquini et al. 2019a). According to our data, at least nine independent introductions of the virus were estimated to have occurred in the CDP area (Fig. 3A and B; Supplementary Table S3). This estimate was consistent with the important genetic diversity of GPGV observed in our parcel $(\pi=0.015 \pm 0.001)$ compared with the overall diversity of the virus in France $(\pi=0.017 \pm 0.002)$. We cannot categorically rule out the possibility that a few grapevines may have been infected at planting; however, because plant replacement has never occurred in this experimental setting, we believe that the significant genetic diversity of the virus was most likely due to the introduction of GPGV in the experimental vineyard from outside sources in association with active vector-mediated transmission of the virus. These introductions most likely resulted from infected vines, as previously observed in
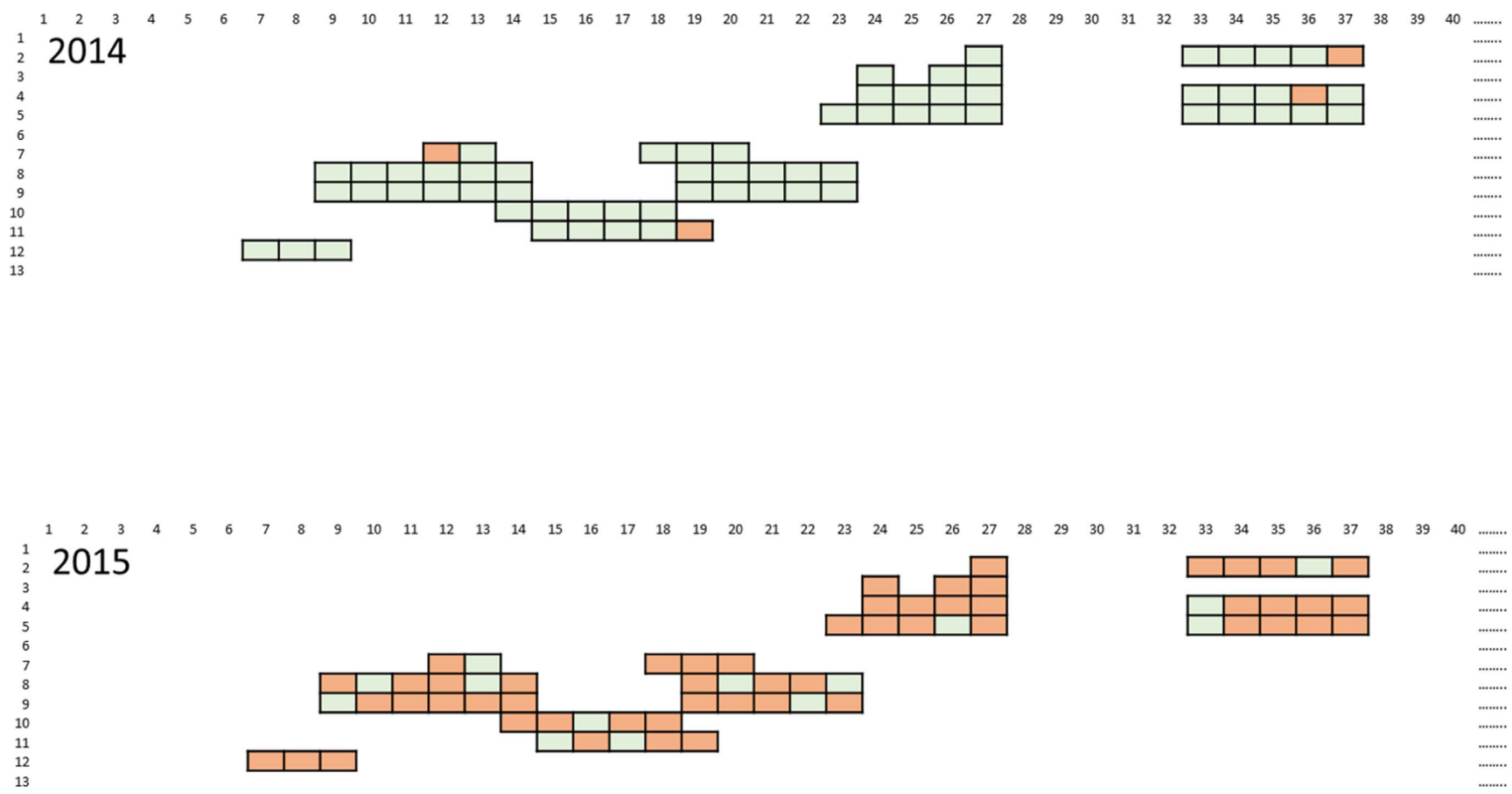

Fig. 4. Evolution of grapevine Pinot gris virus (GPGV) infection status in 67 grapevines from a vineyard in the Châteauneuf-du-Pape region, France, in 2014 and 2015. Squares represent the vine locations in the parcel, and colors represent the status of infection for GPGV tested via reversetranscription PCR (orange = positive and green = negative). 
other regions of the world (Al Rwahnih et al. 2016; Fajardo et al. 2017; Wu and Habili 2017). Introduction of infected planting materials may occur at any time during the renewal of old vineyards or replacement of single plants. In satellite images, significant modifications were quite visible, with many new vineyard planting near our study parcel (within 500 m), especially between 2007 and 2009 (Supplementary Figs. S5 and S6). These observations are not proof of introduction of the virus in the CDP vineyard, although the associated dates are concomitant with those provided by our model prediction, rendering them more realistic. In addition, and from the same in silico analyses, the phylodynamic reconstruction identified two major events of transmission within the vineyard itself, one in 2009 to 2010 and a second in 2014 to 2015 . The second major event of transmission was confirmed after testing 67 grapevines by RT-PCR. Indeed, almost $75 \%$ of the plants became positive for GPGV in 2015 , from 4 of 67 positive plants in 2014 to 53 of 67 positive plants in 2015. Interestingly, according to our sequencing data, the GPGV variants present in the four positive plants in 2014 did not account for the complete genetic diversity of the virus detected in 2017. This result suggested the possible occurrence of transmission not only from within the vineyard but also from surrounding vineyards.

Our results seem to be quite different from those previously described in two vineyards in Italy that also show fast but regular and progressive spread of the virus over time (Bertazzon et al. 2020b). Indeed, by further testing negative grapevines in the following years, our analysis showed that, after these events of transmission, viral progression was limited. In fact, only a marginal number of plants $(6 \%)$ became GPGV positive postoutbreak, with $12 \%$ of the plants remaining negative for the virus over 5 years, although all of them were adjacent to multiple GPGV-positive plants (Fig. 4), demonstrating limited, if any, virus spread after the 2015 outbreak, even under higher viral pressure. This observation raises questions regarding population dynamics of the vector or vectors, the environmental and climatic conditions, and possible cultural changes (i.e., phytosanitary treatments and so on) that may be required for the spread of the virus to occur in the field.

Moreover, we also demonstrated the great genetic stability of GPGV over time, with little to no mismatch detected via Sanger sequencing (Supplementary Table S5). GPGV variants found in the CDP vineyard did not emerge due to virus evolution within the plant itself but, rather, seem to be the result of multiple (simultaneous or sequential) introductions. Furthermore, direct Sanger sequencing did not uncover multivariant infection in plants, which was fully documented via the HTS-based method, but, rather, revealed the presence of the main variant from that plant (main variant defined by the RPKM-based approach from HTS analyses). This observation may mitigate conclusions drawn from previous studies based on RT-PCR and direct Sanger sequencing (without cloning steps), especially those correlating symptomatology with the presence or absence of specific, symptomatic or asymptomatic variants of GPGV.

Overall, our study underlines the fact that many variants might have been present within the close vicinity of our experimental parcel prior to or at the time of planting, and these variants were the probable sources of contamination. We showed that grapevine proximity does not seem to be the main driver of the transmission of specific variants, because variants seem to be scattered randomly throughout the vineyard (Fig. 2B). This distribution pattern raises questions regarding the involvement of $C$. vitis in the spread of GPGV and could fit either of the two following theories. (i) C. vitis is the sole vector of GPGV and is randomly dispersing in the vineyard. Indeed, eriophyids, the smallest known arthropods ( 0.1 to $0.5 \mathrm{~mm}$ ), are known to display efficient passive aerial dispersal behavior (Sabelis and Bruin 1996), with the direction of movement being determined by external forces (i.e., wind, rain, human activity, or phoretic transport). (ii) The distribution pattern of GPGV variants highlights the possible involvement of other vectors, because genetic differentiation in the $C$. vitis population has been demonstrated on a local scale, illustrating the limited movement of mites even within a vineyard (Carew et al. 2004), which could be contradicted by our findings.

Conclusion. From this work, we can conclude that the spread of GPGV can be explosive at the local scale. In addition, even under high viral pressure, some plants (12\% of the 67 vines tested) remained negative over five consecutive years post outbreak (2014 to 2015). This suggests that specific conditions probably need to be met for the spread of the virus to occur and the infection to take place. These conditions are currently unknown; however, vector species and populations and their transmission efficiency in relation to natural environmental conditions may be in question. This is in agreement with some studies showing an apparent lack of natural transmission (Al Rwahnih et al. 2016; Wu and Habili 2017), whereas other studies demonstrated active transmission in vineyards (this study) (Bertazzon et al. 2020b). Together with previous results (Bertazzon et al. 2020b), our findings demonstrated differential spread of GPGV in distinct vineyard locations and, therefore, different environments and ecosystems. To better manage GPGV spread, more work on potential vectors is needed, as has been demonstrated for another emerging grapevine virus and the grapevine red blotch virus (Cieniewicz et al. 2019).

\section{ACKNOWLEDGMENTS}

We thank F. Thevenot for technical support and yearly sampling; the IRD i-Trop HPC, located in Montpellier, for providing HPC resources that have contributed to the research results reported in this article (https://bioinfo.ird.fr/); the bioinformatic team of Rustenholz C. based at INRAE-Grand Est-Colmar; and the reviewers who dedicated their considerable time and expertise to improve this article.

\section{LITERATURE CITED}

Al Rwahnih, M., Golino, D., and Rowhani, A. 2016. First report of grapevine Pinot gris virus infecting grapevine in the United States. Plant Dis. 100:1030. Ayres, D. L., Darling, A., Zwickl, D. J., Beerli, P., Holder, M. T., Lewis, P. O., Huelsenbeck, J. P., Ronquist, F., Swofford, D. L., Cummings, M. P., Rambaut, A., and Suchard, M. A. 2011. BEAGLE: An application programming interface and high-performance computing library for statistical phylogenetics. Syst. Biol. 61:170-173.

Bertazzon, N., Chitarra, W., Angelini, E., and Nerva, L. 2020a. Two new putative plant viruses from wood metagenomics analysis of an esca diseased vineyard. Plants Basel 9:835.

Bertazzon, N., Filippin, L., Forte, V., and Angelini, E. 2016. Grapevine Pinot gris virus seems to have recently been introduced to vineyards in Veneto, Italy. Arch. Virol. 161:711-714.

Bertazzon, N., Forte, V., and Angelini, E. 2020b. Fast transmission of grapevine 'Pinot gris' virus (GPGV) in vineyard. Vitis 59:29-34.

Bertazzon, N., Forte, V., Filippin, L., Causin, R., Maixner, M., and Angelini, E. 2017. Association between genetic variability and titre of grapevine Pinot gris virus with disease symptoms. Plant Pathol. 66:949-959.

Bertazzon, N., Maixner, M., Filippin, L., Bazzo, I., Forte, V., and Angelini, E. 2015. Survey on a new emergent grapevine disease and grapevine Pinot gris virus (GPGV) in Veneto, Northeast Italy. Pages 201-202 in: 18th Congr. ICVG, Ankara, Turkey.

Beuve, M., Candresse, T., Tannières, M., and Lemaire, O. 2015. First report of grapevine Pinot gris virus (GPGV) in grapevine in France. Plant Dis. 99:293.

Bianchi, G. L., De Amicis, F., De Sabbata, L., Di Bernardo, N., Governatori, G., Nonino, F., Prete, G., Marrazzo, T., Versolatto, S., and Frausin, C. 2015. Occurrence of grapevine Pinot gris virus in Friuli Venezia Giulia (Italy): Field monitoring and virus quantification by real-time RT-PCR. Bull. OEPP/ EPPO Bull. 45:22-32.

Carew, M. E., Goodisman, M. A. D., and Hoffmann, A. A. 2004. Species status and population genetic structure of grapevine eriophyoid mites. Entomol. Exp. Appl. 111:87-96. 
Cieniewicz, E., Flasco, M., Brunelli, M., Onwumelu, A., Wise, A., and Fuchs, M. F. 2019. Differential spread of Grapevine red blotch virus in California and New York vineyards. Phytobiomes J. 3:203-211.

Demián, E., Czotter, N., and Várallyay, É. 2018. Detection of Grapevine 'Pinot gris' virus in different non-Vitis hosts in Hungary. In: Proc. 19th Congr. Int. Counc. Study Virus Virus-Like Dis. Grapevine (ICVG), ICVG, Santiago, Chile.

Diaz-Lara, A., Brisbane, R. S., Aram, K., Golino, D., and Al Rwahnih, M. 2019. Detection of new vitiviruses infecting grapevine in California. Arch. Virol. 164:2573-2580.

Drummond, A. J., Ho, S. Y. W., Phillips, M. J., and Rambaut, A. 2006. Relaxed phylogenetics and dating with confidence. PLoS Biol. 4:e88.

Drummond, A. J., Suchard, M. A., Xie, D., and Rambaut, A. 2012. Bayesian phylogenetics with BEAUti and the BEAST 1.7. Mol. Biol. Evol. 29:19691973.

Duchêne, D. A., Duchêne, S., Holmes, E. C., and Ho, S. Y. W. 2015. Evaluating the adequacy of molecular clock models using posterior predictive simulations. Mol. Biol. Evol. 32:2986-2995.

Fajardo, T. V. M., Eiras, M., and Nickel, O. 2017. First report of grapevine Pinot gris virus infecting grapevine in Brazil. Australas. Plant Dis. Notes 12:45.

Firth, C., Kitchen, A., Shapiro, B., Suchard, M. A., Holmes, E. C., and Rambaut, A. 2010. Using time-structured data to estimate evolutionary rates of double-stranded DNA viruses. Mol. Biol. Evol. 27:2038-2051.

Fuchs, M. 2020. Grapevine viruses: A multitude of diverse species with simple but overall poorly adopted management solutions in the vineyard. J. Plant Pathol. 102:643-653.

Garcia, S., Hily, J.-M., Komar, V., Gertz, C., Demangeat, G., Lemaire, O., and Vigne, E. 2019. Detection of multiple variants of grapevine fanleaf virus in single Xiphinema index nematodes. Viruses 11:1139.

Giampetruzzi, A., Roumi, V., Roberto, R., Malossini, U., Yoshikawa, N., La Notte, P., Terlizzi, F., Credi, R., and Saldarelli, P. 2012. A new grapevine virus discovered by deep sequencing of virus- and viroid-derived small RNAs in cv Pinot gris. Virus Res. 163:262-268.

Gill, M. S., Lemey, P., Faria, N. R., Rambaut, A., Shapiro, B., and Suchard, M. A. 2012. Improving Bayesian population dynamics inference: A coalescent-based model for multiple loci. Mol. Biol. Evol. 30:713-724.

Gualandri, V., Asquini, E., Bianchedi, P., Covelli, L., Brilli, M., Malossini, U., Bragagna, P., Saldarelli, P., and Si-Ammour, A. 2017. Identification of herbaceous hosts of the grapevine Pinot gris virus (GPGV). Eur. J. Plant Pathol. 147:21-25.

Hily, J.-M., Demanèche, S., Poulicard, N., Tannières, M., Djennane, S., Beuve, M., Vigne, E., Demangeat, G., Komar, V., Gertz, C., Marmonier, A., Hemmer, C., Vigneron, S., Marais, A., Candresse, T., Simonet, P., and Lemaire, O. 2018. Metagenomic-based impact study of transgenic grapevine rootstock on its associated virome and soil bacteriome. Plant Biotechnol. J. 16:208-220.

Hily, J.-M., Poulicard, N., Candresse, T., Vigne, E., Beuve, M., Renault, L., Velt, A., Spilmont, A.-S., and Lemaire, O. 2020. Datamining, genetic diversity analyses and phylogeographic reconstructions redefine the worldwide evolutionary history of grapevine Pinot gris virus and grapevine berry inner necrosis virus. Phytobiomes J. 4:165-177.

Kumar, S., Stecher, G., Li, M., Knyaz, C., and Tamura, K. 2018. MEGA X: Molecular evolutionary genetics analysis across computing platforms. Mol. Biol. Evol. 35:1547-1549.

Kunugi, Y., Asari, S., Terai, Y., and Shinkai, A. 2000. Studies on the grapevine berry inner necrosis virus disease, 2: Transmission of grapevine berry inner necrosis virus by the grape erineum mite, Colomerus vitis in Yamanashi. Bull. Yamanashi Fruit Tree Exp. Stn. Jpn. 10:57-63.

Lemey, P., Rambaut, A., Drummond, A. J., and Suchard, M. A. 2009. Bayesian phylogeography finds its roots. PLOS Comput. Biol. 5:e1000520.

Malagnini, V., de Lillo, E., Saldarelli, P., Beber, R., Duso, C., Raiola, A., Zanotelli, L., Valenzano, D., Giampetruzzi, A., Morelli, M., Ratti, C., Causin, R., and Gualandri, V. 2016. Transmission of grapevine Pinot gris virus by Colomerus vitis (Acari: Eriophyidae) to grapevine. Arch. Virol. 161:2595-2599.

Malossini, U., Bianchedi, P., Beber, R., Credi, R., Saldarelli, P., and Gualandri, V. 2015. Spread of GPGV-associated disease in two vineyards in Trentino (Italy). Pages 212-213 in: 18th Congress of ICVG, Ankara, Turkey.
Marra, M., Giampetruzzi, A., Abou Kubaa, R., de Lillo, E., and Saldarelli, P. 2020. Grapevine Pinot gris virus variants in vines with chlorotic mottling and leaf deformation. J. Plant Pathol. 102:531.

Martelli, G. P. 2017. An overview on grapevine viruses, viroids, and the diseases they cause. Pages 31-46 in: Grapevine Viruses: Molecular Biology, Diagnostics and Management. B. Meng, G. P. Martelli, D. A. Golino, and M. Fuchs, eds. Springer International Publishing, Cham, Switzerland.

Minin, V. N., and Suchard, M. A. 2008a. Counting labeled transitions in continuous-time Markov models of evolution. J. Math. Biol. 56:391-412.

Minin, V. N., and Suchard, M. A. 2008b. Fast, accurate and simulation-free stochastic mapping. Philos. Trans. R. Soc. B. 363:3985-3995.

Morán, F., Olmos, A., Lotos, L., Predajňa, L., Katis, N., Glasa, M., Maliogka, V., and Ruiz-García, A. B. 2018. A novel specific duplex real-time RT-PCR method for absolute quantitation of grapevine Pinot gris virus in plant material and single mites. PLoS One 13:e0197237.

Murray, G. G. R., Wang, F., Harrison, E. M., Paterson, G. K., Mather, A. E., Harris, S. R., Holmes, M. A., Rambaut, A., and Welch, J. J. 2016. The effect of genetic structure on molecular dating and tests for temporal signal. Methods Ecol. Evol. 7:80-89.

Nourinejhad Zarghani, S., Hily, J. M., Glasa, M., Marais, A., Wetzel, T., Faure, C., Vigne, E., Velt, A., Lemaire, O., Boursiquot, J. M., Okic, A., RuizGarcia, A. B., Olmos, A., Lacombe, T., and Candresse, T. 2018. Grapevine virus $\mathrm{T}$ diversity as revealed by full-length genome sequences assembled from high-throughput sequence data. PLoS One 13:e0206010.

Pleško, I. M., Marn, M. V., Seljak, G., and Žežlina, I. 2014. First report of grapevine Pinot gris virus infecting grapevine in Slovenia. Plant Dis. 98:1014.

Poojari, S., Lowery, T., Rott, M., Schmidt, A.-M., and Úrbez-Torres, J. R. 2016. First report of grapevine Pinot gris virus in British Columbia, Canada. Plant Dis. 100:1513.

Rambaut, A., Lam, T. T., Max Carvalho, L., and Pybus, O. G. 2016. Exploring the temporal structure of heterochronous sequences using TempEst (formerly Path-O-Gen). Virus Evol. 2:vew007.

Renault-Spilmont, A.-S., Sevin Preyre, A.-F., Jérémy, G., Beuve, M., Alliaume, A., Marais, A., Faure, C., Candresse, T., and Lemaire, O. 2018. Occurrence of grapevine Pinot gris virus (GPGV) and grapevine leaf mottling and deformation (GLMD) syndrome in France: Genetic diversity and field monitoring in diverse viticulture areas. In: Proc. 19th Congr. Int. Counc. Study Virus Virus-Like Dis. Grapevine (ICVG), ICVG, Santiago, Chile.

Reynard, J. S., Schumacher, S., Menzel, W., Fuchs, J., Bohnert, P., Glasa, M., Wetzel, T., and Fuchs, R. 2016. First report of grapevine Pinot gris virus in German vineyards. Plant Dis. 100:2545.

Ruiz-García, A. B., and Olmos, A. 2017. First report of grapevine Pinot gris virus in grapevine in Spain. Plant Dis. 101:1070.

Sabelis, M. W., and Bruin, J. 1996. 1.5.3. Evolutionary ecology: Life history patterns, food plant choice and dispersal. Pages 329-366 in: World Crop Pests, Vol. 6. E. E. Lindquist, M. W. Sabelis, and J. Bruin, eds. Elsevier, Amsterdam, The Netherlands.

Saldarelli, P., Giampetruzzi, A., Morelli, M., Malossini, U., Pirolo, C., Bianchedi, P., and Gualandri, V. 2015. Genetic variability of grapevine Pinot gris virus and its association with grapevine leaf mottling and deformation. Phytopathology 105:555-563.

Tarquini, G., De Amicis, F., Martini, M., Ermacora, P., Loi, N., Musetti, R., Bianchi, G. L., and Firrao, G. 2019a. Analysis of new grapevine Pinot gris virus (GPGV) isolates from Northeast Italy provides clues to track the evolution of a newly emerging clade. Arch. Virol. 164: 1655-1660.

Tarquini, G., Zaina, G., Ermacora, P., De Amicis, F., Franco-Orozco, B., Loi, N., Martini, M., Bianchi, G. L., Pagliari, L., Firrao, G., de Paoli, E., and Musetti, R. 2019b. Agroinoculation of grapevine Pinot gris virus in tobacco and grapevine provides insights on viral pathogenesis. PLoS One 14: e0214010.

Vigne, E., Garcia, S., Komar, V., Lemaire, O., and Hily, J.-M. 2018. Comparison of serological and molecular methods with high-throughput sequencing for the detection and quantification of grapevine fanleaf virus in vineyard samples. Front. Microbiol. 9:2726.

Wu, Q., and Habili, N. 2017. The recent importation of grapevine Pinot gris virus into Australia. Virus Genes 53:935-938. 